\title{
The Effect of Defect Disorder on the Electronic Structure of Rutile $\mathrm{TiO}_{2-\mathrm{x}}$
}

\author{
Faruque M. Hossain ${ }^{1), a)}$ and G. E. Murch \\ Diffusion in Solids Group, School of Engineering, The University of Newcastle, Callaghan, \\ NSW 2308, Australia.
}

and

\section{Sheppard and J. Nowotny}

\author{
Centre for Materials Research in Energy Conversion, School of Materials Science and \\ Engineering, The University of New South Wales, NSW 2052, Australia.
}

Keywords: Electronic structure, titanium oxide, defect formation energies, nonstoichiometry

\begin{abstract}
The purpose of this work is to study the effect of bulk point defects on the electronic structure of rutile $\mathrm{TiO}_{2}$. The paper is focused on the effect of oxygen nonstoichiometry in the form of oxygen vacancies, Ti interstitials and $\mathrm{Ti}$ vacancies and related defect disorder on the band gap width and on the local energy levels inside the band gap. Ab initio density functional theory is used to calculate the formation energies of such intrinsic defects and to detect the positions of these defect induced energy levels in order to visualize the tendency of forming local mid-gap bands. Apart from the formation energy of the Ti vacancies (where experimental data do not exist) our calculated results of the defect formation energies are in fair agreement with the experimental results and the defect energy levels consistently support the experimental observations. The calculated results indicate that the exact position of defect energy levels depends on the estimated band gap and also the charge state of the point defects of $\mathrm{TiO}_{2}$.
\end{abstract}

\section{Introduction}

The electronic structure of point defects in oxide semiconductors has been extensively studied in order to establish the relationship between defect chemistry and semi-conducting properties. In principle, a perfect stoichiometric metal oxide exhibits insulating properties. Preparation-induced intrinsic defects or structural changes can turn these oxide materials into semiconducting or conducting depending on the concentration of defects introduced or the level of structural deformation. Point defects in oxide materials either form deep energy levels, which may act as trapping centers or shallow energy levels, which may act as donors. In order to understand the effect of defect disorder on the electronic properties, it is essential to know the energetic position of the various defects inside the band gap which can predominate.

Titanium dioxide $\left(\mathrm{TiO}_{2}\right)$ is a wide-gap semiconductor and important oxide material because of its broad range of technological applications, long-term chemical stability, and non-toxicity. Since the pioneering work of Fujishima and Honda [1], $\mathrm{TiO}_{2}$ has received special attention as a prime candidate material for photo-electrochemical water-splitting and other photo-catalytic applications [2-4]. Titanium dioxide is a promising semiconducting material on account of its chemical stability in aqueous environments and under high energy illumination [3]. However, due to its large band gap, $\sim 3-3.2 \mathrm{eV}$ (for rutile and anatase phases respectively), titanium dioxide lacks 
sensitivity to visible light, which is necessary for high performance under solar illumination. This is especially the case if commercial efficiencies are to be achieved for the generation of hydrogen from water [3]. Hence, efforts have been made to increase the visible light sensitivity through band gap reduction $[3,4]$ or by introducing defect energy levels in the form of intermediate bands. However, an understanding of how the band gap can be manipulated and how an intermediate defect bands can be formed in practice is limited.

The possibility of band gap reduction using extrinsic doping with lattice matched foreign atoms has been reported in the literature, but those doped materials suffer from thermal instability and introduced higher carrier recombination centers. Such extrinsic doping techniques do not satisfactorily increase the efficiency of photo-electrochemical water-splitting and other photocatalytic devices. It is instructive in this context to note that intrinsic point defects in the bulk and surface of $\mathrm{TiO}_{2}$ may introduce defect energy levels inside the band gap. Moreover, larger aggregates of point defect clusters may form energy bands inside the band gap, which in turn may enhance the quantum efficiency of appropriate devices. It is also a matter of concern that the quantum efficiency alone cannot increase the overall efficiency of these devices. Moreover, it is necessary to increase the conductivity of the electrode material made of $\mathrm{TiO}_{2}$ as well. $\mathrm{Pure}_{\mathrm{TiO}_{2}}$ at room temperature is an electrical insulator. It is well known that the electrical conductivity of $\mathrm{TiO}_{2}$ varies with the dopant types, $\mathrm{O} / \mathrm{Ti}$ atomic ratio, and its preparation conditions (thermal treatment and variation in oxygen partial pressure). In the case of reduced rutile $\mathrm{TiO}_{2-\mathrm{x}}$, published research suggests that oxygen vacancies and $\mathrm{Ti}^{3+}$ interstitials may be the important defects in the system. Few reports suggest the dominance of Ti vacancies.

Experimental results of Cronemeyer [5] and Ghosh et al. [6] systematically represent the effect of intrinsic point defects in forming local energy levels inside the band gap of rutile $\mathrm{TiO}_{2}$. Based on these experimental results, we make an illustration as shown in Fig. 1, which indicates the energy levels of defect states associated with the oxygen vacancies and Ti interstitials, in order to compare our theoretical results of point defects. Infrared absorption spectroscopy of Cronemeyer showed that the oxygen vacancies produce two energy levels at $0.75 \mathrm{eV}$ and $1.18 \mathrm{eV}$ (Fig. 1(a)) below the conduction band edge, which are singly $\left(V_{o}^{\mathrm{g}}\right)$ and doubly $\left(V_{o}^{\mathrm{g}}\right)$ ionized respectively. On the other hand, Ghosh et al. identified eight $(0.27,0.28,0.39,0.48,0.56,0.62,0.76$, and $0.87 \mathrm{eV}$ below the conduction band edge) discrete energy levels on a slightly reduced rutile $\mathrm{TiO}_{2}$ sample. They proposed that the energy level at $0.76 \mathrm{eV}$ is responsible for the singly ionized O-vacancy $\left(V_{o}^{\mathrm{g}}\right)$ when compared with the results of Cronemeyer [5]. 
(a)

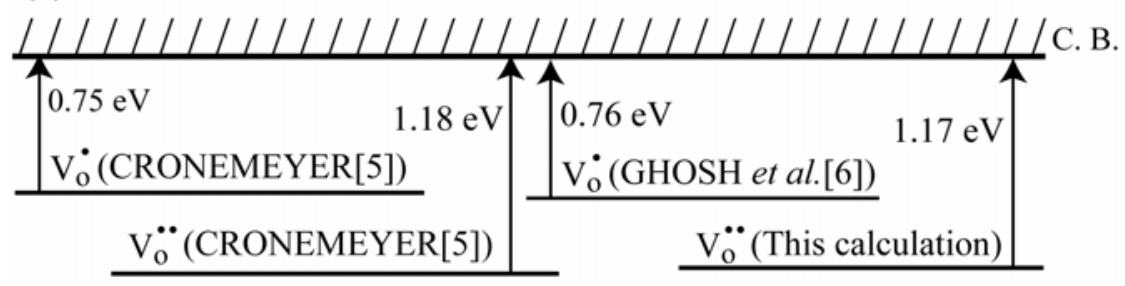

mid-gap

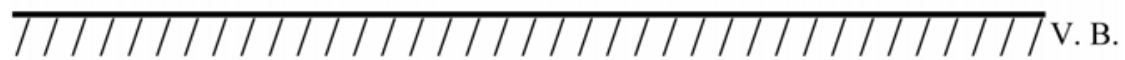

(b)

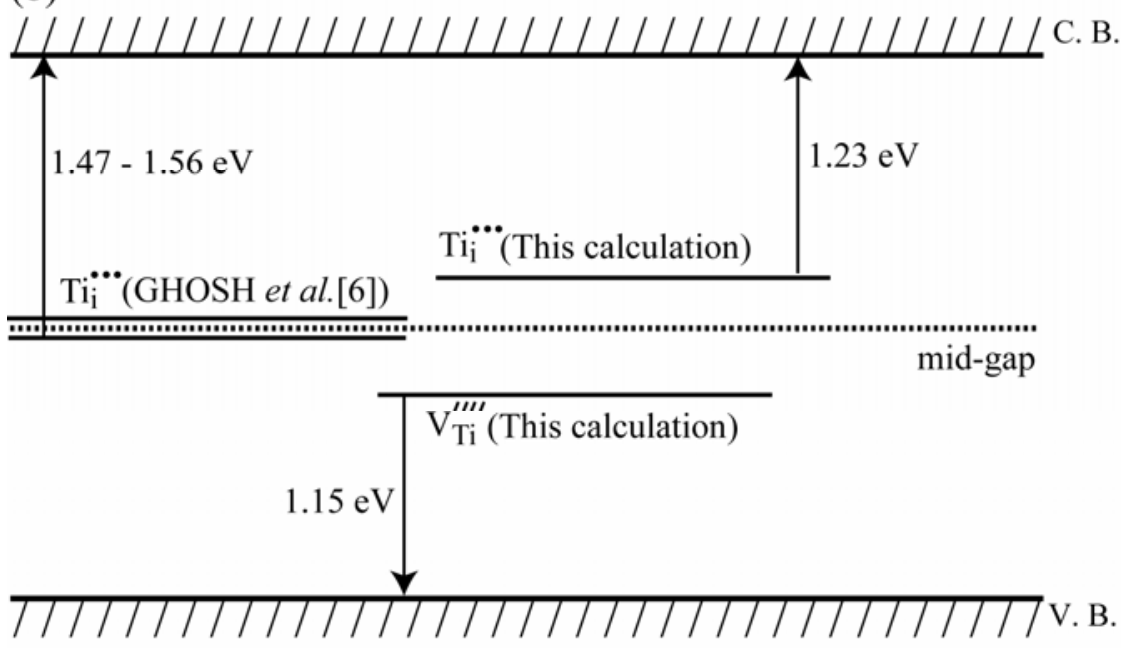

Fig. 1: Defect induced energy level (experimental and the present calculations) diagram of bulk rutile $\mathrm{TiO}_{2}$. (a) Oxygen vacancy induced defect energy diagram (b) Interstitial and vacancy $\mathrm{Ti}$ induced defect energy diagram.

The origin of other discrete shallow levels was unidentified. They also detected a narrow band at 1.47 - $1.56 \mathrm{eV}$ (Fig. 1(b)) below the conduction band and tentatively identified its presence as due to interstitial $\mathrm{Ti}^{3+}$ ions. Hence, theoretical investigation is necessary to identify the experimentally observed defect energy levels inside the band gap of reduced rutile $\mathrm{TiO}_{2}$. There are few theoretical attempts that have been made in order to support the experimental results.

Munnix et al. [7] have calculated the role of bulk oxygen vacancies in rutile $\mathrm{TiO}_{2}$ by a scattering theoretical method using a classical Green's function. They found that bulk oxygen vacancies make no contribution to induce any defect states inside the band gap of $\mathrm{TiO}_{2}$. In contrast, Ramamoorthy et al. [8] and Yu and Halley [9] found that oxygen vacancies and $\mathrm{Ti}$ interstitials introduce defect energy levels inside the band gap. These results are tabulated here (Table 1) in order to compare our theoretical results with different methods of calculation. It is recognized that the exact defect energy levels can not be identified unless the calculated band gap is exactly reproduced to the experimentally observed band gap. This is probably the reason for several different energy levels of oxygen vacancy and Ti interstitial represented in Table 1 for different method of calculations. Yu and Halley [9] estimated the band gap and the energy levels of both single and double O-vacancies and $\mathrm{Ti}$ interstitials in the rutile $\mathrm{TiO}_{2}$ structure to be close to the experimental results of Cronemeyer [5]. However, $\mathrm{Yu}$ and Halley did not calculate the formation 
energy of oxygen vacancy and Ti interstitial in their model. It is worth mentioning that the calculated defect energy levels will be more reliable when the formation energy of defects is consistent with experimental observation.

\begin{tabular}{|c|c|c|c|c|}
\hline $\begin{array}{l}\text { Method of } \\
\text { calculation }\end{array}$ & $\begin{array}{c}\text { Band gap, } \\
E_{g}(\mathrm{eV})\end{array}$ & $\begin{array}{c}E_{c}-E\left(V_{o}^{\bullet \bullet}\right) \\
(\mathrm{eV})\end{array}$ & $E_{c}-E\left(T i_{i}^{\bullet \bullet \bullet}\right)(\mathrm{eV})$ & $E\left(V_{T i}\right)-E_{v}(\mathrm{eV})$ \\
\hline $\begin{array}{l}\text { Self-consistent } \\
\text { seudopotential } \\
\text { total-energy } \\
\text { calculation [Ref. 8] }\end{array}$ & $\sim 2.0$ & 0.3 & --- & ---- \\
\hline $\begin{array}{l}\text { Semiempirical self- } \\
\text { consistent method } \\
\text { [Ref. 9] }\end{array}$ & $\sim 3.3$ & $\begin{array}{c}0.7 \\
\text { (when one } \\
\text { oxygen atom is } \\
\text { removed) }\end{array}$ & $\begin{array}{l}\text { 0.19, } 0.8 \\
\text { (when one Ti atom is } \\
\text { inserted in a unit cell) }\end{array}$ & --- \\
\hline & & $\begin{array}{c}0.22,1.28 \\
\text { (when two } \\
\text { oxygen atoms } \\
\text { are removed) }\end{array}$ & $\begin{array}{c}\text { 0.35, } 0.74,0.95 \\
\text { (when two Ti atoms } \\
\text { are inserted in two } \\
\text { neighboring unit } \\
\text { cells) }\end{array}$ & \\
\hline $\begin{array}{l}\text { Density functional } \\
\text { total energy } \\
\text { calculation [This } \\
\text { calculation] }\end{array}$ & $\sim 3.0$ & 1.17 & $\begin{array}{c}1.23 \\
2.14 \\
\text { (Octahedral } \\
\text { distortion) }\end{array}$ & 1.15 \\
\hline
\end{tabular}

Table 1: Oxygen vacancy, Ti interstitial, and Ti vacancy induced energy levels inside the band gap of bulk rutile $\mathrm{TiO}_{2}$ for different methods of calculation.

In order to best reproduce experimental results, the formation energies of $\mathrm{Ti}$ interstitials, oxygen vacancies, and $\mathrm{Ti}$ vacancies in the bulk rutile $\mathrm{TiO}_{2}$ should be calculated using the first principles total energy density functional (DFT) method. The determination of the local energy levels by these defects inside the band gap will then be reliable. Such information related to Ti vacancies has not previously been determined. 


\section{Method of Calculation}

A $2 a \times 2 b \times 2 c$ super cell was used in this calculation as a model for bulk rutile $\mathrm{TiO}_{2}$. The approach of the calculation is based on density functional theory (DFT) within the generalized gradient approximation (GGA-PBE) proposed by Perdew et al. [10] and employs Vanderbilt ultrasoft pseudopotentials (USP)[11] for electron-ion interaction. Electron wave functions were expanded in terms of a plane wave basis set, and the electronic ground state was reached using selfconsistent electronic minimization as implemented by the computer code CASTEP $[12,13]$. An ultrasoft pseudopotential for Ti and $\mathrm{O}$ was produced from the configurations [Ne]3s $3 p^{6} 3 d^{2} 4 s^{2}$ and [He] $2 s^{2} 2 p^{4}$, respectively. We used $4 k$-points Brillouin-zone sampling for geometry optimization and $8 k$-points for density of states calculations. In both the calculations the plane-wave cutoff energy of $300 \mathrm{eV}$ was chosen. All the atoms in the $2 a \times 2 b \times 2 c$ defect-free super cell were relaxed from their initial positions with energy convergence tolerance of $2.0 \times 10^{-5} \mathrm{eV} / \mathrm{atom}$ and a maximum force of $0.05 \mathrm{eV} / \AA$. A similar convergence tolerance for energy and maximum force was used for the atomic relaxation of defect-containing structures.

\section{Stoichiometric superstructure}

The $2 a \times 2 b \times 2 c$ super cell $\left(\mathrm{Ti}_{16} \mathrm{O}_{32}\right)$ is used as a stoichiometric $\mathrm{TiO}_{2}$ super-structure. It is considered that the $2 \times 2 \times 2$ unit cell is large enough to create point defects within a reasonable limit of defect concentration. The partial density of states (PDOS) was calculated for the stoichiometric $\mathrm{TiO}_{2}$ super-structure as shown in Fig. 2. It represents the position of the Fermi level, $E_{F}$ at the top of the completely filled valence band, which is comprised mainly of $\mathrm{O} 2 p$ states, and the conduction band, which is comprised mainly of $\mathrm{Ti} 3 d$ sates, is completely empty. The result shows that stoichiometric rutile $\mathrm{TiO}_{2}$ is a perfect insulator. The underestimated band gap (the limitation of GGA) is adjusted towards the experimental value $(3.05 \mathrm{eV})$ using a scissors operator.

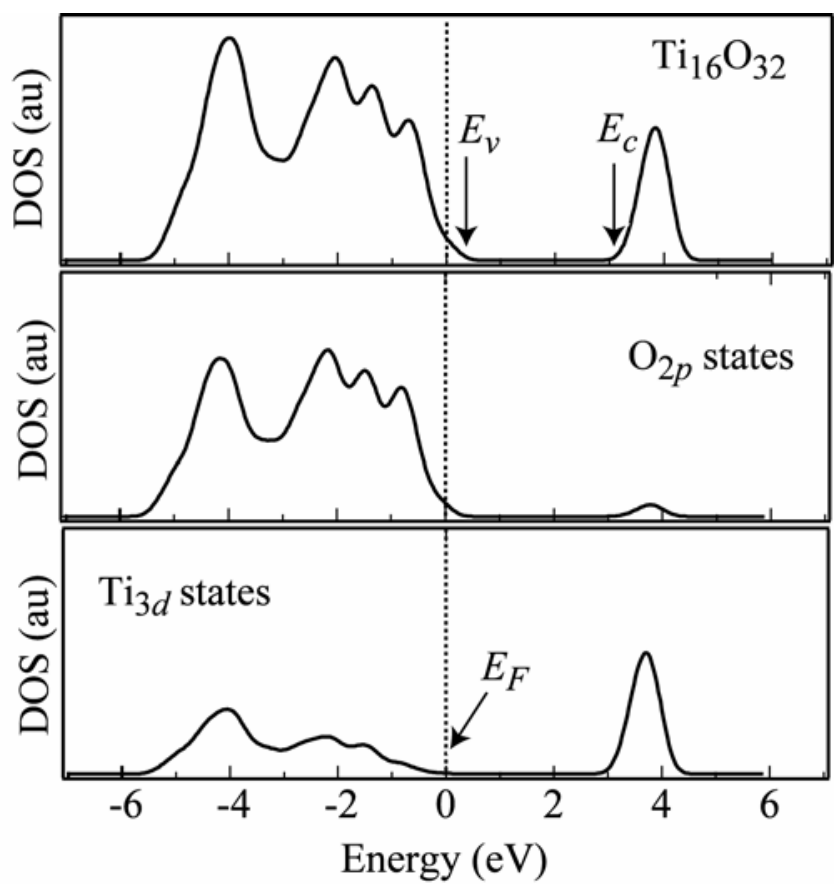

Fig. 2: Density of states (DOS) of stoichiometric bulk rutile $\mathrm{TiO}_{2}$. Zero energy indicates the Fermi level.

It is worth noting that the scissors operator rigidly shifts the unoccupied conduction band with respect to completely occupied valence band, which virtually represents the excitation energy of optical transition. There are no gap states found inside the band gap of stoichiometric rutile $\mathrm{TiO}_{2}$. 
On the other hand, partial DOS of $\mathrm{O} 2 p$ and $\mathrm{Ti} 3 d$ show $p$-d hybridization. This means that stoichiometric rutile $\mathrm{TiO}_{2}$ is not a perfect ionic crystal, rather, it shows coexistence of both ionic and covalency characters.

\section{Nonstoichiometric superstructure}

A model structure of $\mathrm{Ti}$ interstitial $\left(T i_{i}\right)$ was prepared by inserting a $\mathrm{Ti}$ atom with a reduced oxidation state $\left(\mathrm{Ti}^{3+}\right)$ at the $\left(\frac{3 a}{2}, b, c\right)$ fractional position in a vacant octahedral site of the supercell. Six oxygen atoms surrounding the Ti interstitial were bonded to form new octahedra. These seven octahedral atoms were allowed to relax through the energy minimization while all other atoms in the supercell were constrained (fixed) to their original fractional positions. A model of an oxygen vacancy was made by removing an $\mathrm{O}$ atom from the octahedra. The central $\mathrm{Ti}$ atom of the octahedra was located at $\left(\frac{a}{2}, \frac{b}{2}, \frac{c}{2}\right)$ fractional position in the supercell. Three Ti atoms, which were bonded with the removed oxygen atom, were allowed to relax through the energy minimization while all other atoms in the supercell remained constrained to their original fractional positions. To model a doubly ionized oxygen vacancy $\left(V_{o}^{\text {g }}\right)$, which is equivalent to a model of O-vacancy $-2 \mathrm{Ti}^{3+}$ complex, in this structure we modified the formal charge of two Ti atoms among those three from $+4 e$ to $+3 e$, which in turn make the charge of the structure neutral. Similarly, a model for a $\mathrm{Ti}$ vacancy was formed by removing a Ti atom from its $(a, b, c)$ fractional position in the supercell $\left(\mathrm{Ti}_{15} \mathrm{O}_{32}\right)$. All the atoms in the supercell were allowed to relax through energy minimization. The calculation converges within the above mentioned tolerance for energy and maximum force as was used for a defect-free supercell. We also applied the same scissors operator for these three cases as used in the stoichiometric case to adjust the band gap.

\section{Defect formation energies}

The formation energy of $\mathrm{Ti}_{i}$ in the bulk rutile $\mathrm{TiO}_{2}$ can be calculated as follows:

$$
E_{f}^{T i_{i}}=E^{\text {total }}\left(T i_{i}\right)-\left[E^{\text {total }}(\text { bulk })+\mu_{T i}\right]
$$

where $E^{\text {total }}\left(T i_{i}\right)$ is the optimized (relaxed) total energy of $2 \times 2 \times 2$ super cell when one Ti atom is inserted in the super cell $\left(\mathrm{Ti}_{17} \mathrm{O}_{32}\right), E^{\text {total }}$ (bulk) is the relaxed total energy of $2 \times 2 \times 2$ super cell $\left(\mathrm{Ti}_{16} \mathrm{O}_{32}\right)$, and $\mu_{T i}$ is the chemical potential of Ti. However, the chemical potential of $\mathrm{Ti}$ and $\mathrm{O}$ are not independent in the $\mathrm{TiO}_{2}$ system, they are related by

$$
\mu_{T i}+2 \mu_{o}=\mu_{T_{i O}}=E_{t}\left[\mathrm{TiO}_{2}\right]
$$

where $\mu_{o}$ is the chemical potential of an $\mathrm{O}$ atom, and $\mu_{\mathrm{TiO}_{2}}$ is the total energy of a unit cell of $\mathrm{TiO}_{2}$ $\left(E_{t}\left[\mathrm{TiO}_{2}\right]\right)$. However, the calculation of absolute values of $\mu_{T i}$ and $\mu_{o}$ in $\mathrm{TiO}_{2}$ is complicated. Here, we have calculated the limiting values of $\mu_{T i}$ and $\mu_{o}$ in Ti-O systems by calculating the total energy of unit cells for three stable phases $\left(\mathrm{TiO}, \mathrm{Ti}_{2} \mathrm{O}_{3}\right.$, and $\left.\mathrm{TiO}_{2}\right)$ of titanium oxide between Timetal and gaseous (molecular) $\mathrm{O}_{2}$. Hence, the chemical potential of $\mathrm{Ti}$ and $\mathrm{O}$ in the Ti-O system is related by the following equations: 
$\mu_{T i}=E_{t}[T i]$ (for Ti-metal)

$\mu_{T i}+\mu_{o}=\mu_{T i O}=E_{t}[\mathrm{TiO}]$ (for $\mathrm{TiO}$ )

$2 \mu_{T i}+3 \mu_{o}=\mu_{T_{2} \mathrm{O}_{3}}=E_{t}\left[\mathrm{Ti}_{2} \mathrm{O}_{3}\right]$ (for $\mathrm{Ti}_{2} \mathrm{O}_{3}$ )

$2 \mu_{o}=E_{t}\left[O_{2}\right]$ (for $\mathrm{O}_{2}$-molecule)

Simultaneous solution of Eq. (3) and (4) and Eq. (2) and (6) provides the value of $\mu_{T i}$ and $\mu_{o}$ for Tirich and O-rich conditions, respectively. All other limiting values of chemical potentials are illustrated in Fig. 3. Inserting the values of $\mu_{T i}$ in Eq. (1) we calculate the formation energy of an interstitial $\mathrm{Ti}, \mathrm{Ti}_{i}$ for both $\mathrm{Ti}$-rich and $\mathrm{O}$-rich conditions.

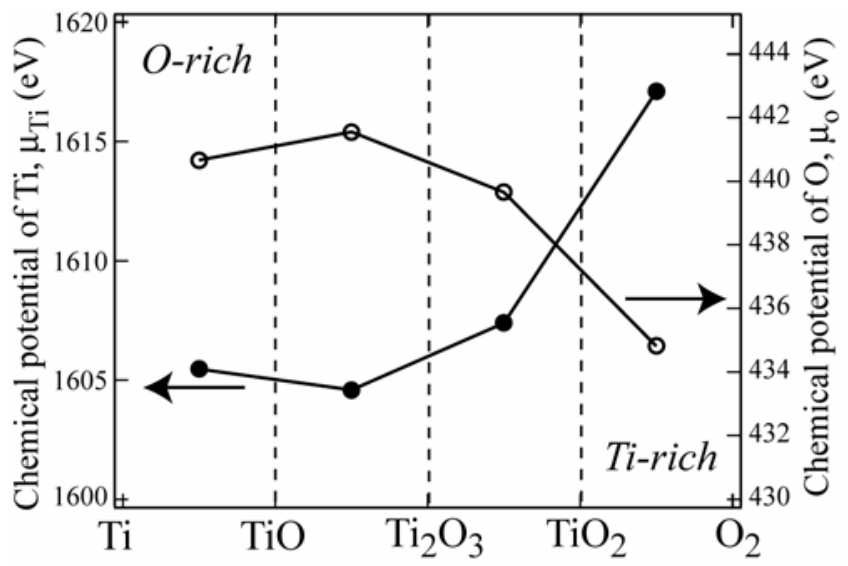

Fig. 3: Calculated limiting values of the chemical potentials of $\mathrm{Ti}$ and $\mathrm{O}$ for three different stable phases between Ti-metal and gaseous (molecular) $\mathrm{O}_{2}$ in the Ti-O system.

The formation energy of an oxygen vacancy in bulk $\mathrm{TiO}_{2}$ can be calculated as follows:

$E_{f}^{V_{o}}=E^{\text {total }}($ bulk $)-\left[E^{\text {total }}\left(V_{o}\right)+\mu_{o}\right]$

where $E^{\text {total }}\left(V_{o}\right)$ is the optimized (relaxed) total energy of the $2 \times 2 \times 2$ supercell in the presence of an oxygen vacancy. Inserting the values of $\mu_{o}$ in Eq. (7) we calculated the formation energy of the oxygen vacancy, $V_{o}$ for both Ti-rich and $O$-rich conditions.

The formation energy of $\mathrm{Ti}$ vacancy in the bulk $\mathrm{TiO}_{2}$ can be calculated as:

$E_{f}^{V_{T i}}=E^{\text {total }}($ bulk $)-\left[E^{\text {total }}\left(V_{T i}\right)+\mu_{T i}\right]$

where $E^{\text {total }}\left(V_{T i}\right)$ is the optimized (relaxed) total energy of the $2 \times 2 \times 2$ super cell with the Ti vacancy. Inserting the values of $\mu_{T i}$ in Eq. (8) we calculatd the formation energy of the Ti vacancy, $V_{T i}$ for both Ti-rich and O-rich conditions. 
Table 2:

Experimentally obtained and calculated formation energy of point defects in bulk rutile $\mathrm{TiO}_{2}$.

\begin{tabular}{|c|c|c|c|c|c|}
\hline $\begin{array}{l}\text { Method of } \\
\text { experiments } \\
\text { and } \\
\text { calculations }\end{array}$ & Temp. (K) & $\mathrm{P}_{\mathrm{O} 2}$ (atm.) & $\begin{array}{r}\text { Defect } \\
\text { Str-re }\end{array}$ & $\begin{array}{c}\text { Formation } \\
\text { energy }(\mathrm{eV})\end{array}$ & Ref \\
\hline $\begin{array}{c}\text { Thermo- } \\
\text { gravimetric } \\
\text { study }\end{array}$ & $860-1050^{\circ} \mathrm{C}$ & $\begin{array}{l}1.71 \times 10^{-2} \\
-6.84 \times 10^{-1}\end{array}$ & $V_{o}$ & $3.9,5.6$ & {$[14,15]$} \\
\hline $\begin{array}{c}\text { Thermo- } \\
\text { gravimetric } \\
\text { study }\end{array}$ & $920-1220^{\circ} \mathrm{C}$ & $\begin{array}{c}1.0 \times 10^{-9} \\
-1.0 \times 10^{-17}\end{array}$ & $V_{o}$ & 5.6 & {$[16]$} \\
\hline \multirow{3}{*}{$\begin{array}{c}\text { Thermo- } \\
\text { gravimetric } \\
\text { and electrical } \\
\text { study }\end{array}$} & \multirow[t]{3}{*}{$800-1100^{\circ} \mathrm{C}$} & \multirow{3}{*}{$\begin{array}{c}1.0 \times 10^{-5} \\
-1.0 \times 10^{-20}\end{array}$} & $T i_{i}^{4+}$ & 10.1 & \multirow[t]{3}{*}{ [17] } \\
\hline & & & $V_{o}^{2+}$ & 4.6 & \\
\hline & & & $V_{o}^{+}$ & $3.6-4.0$ & \\
\hline $\begin{array}{l}\text { Self-diffusion } \\
\text { of }{ }^{44} \mathrm{Ti} \text { in } \\
\text { reduced } \mathrm{TiO}_{2}\end{array}$ & $1000-1100^{\circ} \mathrm{C}$ & $\begin{array}{c}1.0 \times 10^{-13} \\
-1.0 \times 10^{-16}\end{array}$ & $T i_{i}^{4+}$ & $11.3-12.7$ & [18] \\
\hline \multirow{2}{*}{$\begin{array}{c}\text { The polarizable } \\
\text { point ion } \\
\text { shell model }\end{array}$} & \multirow[t]{2}{*}{--} & \multirow[t]{2}{*}{-- } & $\mathrm{Ti}_{i}^{4+}$ & 10.8 & \multirow[t]{2}{*}{ [19] } \\
\hline & & & $V_{o}^{2+}$ & 10.1 & \\
\hline \multirow[t]{2}{*}{$\begin{array}{c}\text { Electrical } \\
\text { conductivity }\end{array}$} & \multirow[t]{2}{*}{$1000-1500^{\circ} \mathrm{C}$} & \multirow[t]{2}{*}{$\begin{array}{c}1.0 \\
-1.0 \times 10^{-15}\end{array}$} & $\mathrm{Ti}_{i}^{3+}$ & 9.24 & \multirow[t]{2}{*}[20]{} \\
\hline & & & $T i_{i}^{4+}$ & 10.67 & \\
\hline \multirow{3}{*}{$\begin{array}{l}\text { Density } \\
\text { functional } \\
\text { total energy } \\
\text { calculation }\end{array}$} & \multirow[t]{3}{*}{--} & \multirow[t]{3}{*}{--} & $T i_{i}^{3+}$ & $\begin{array}{c}0.06 \text { (Ti-rich); } \\
11.7 \text { (O-rich) }\end{array}$ & \multirow[t]{3}{*}{$\begin{array}{l}\text { This } \\
\text { work }\end{array}$} \\
\hline & & & $V_{o}^{2+}$ & $\begin{array}{c}0.18 \text { (Ti-rich); } \\
6.02 \text { (O-rich) }\end{array}$ & \\
\hline & & & $V_{T i}^{4-}$ & $\begin{array}{c}13.96 \text { (Ti-rich); } \\
2.32 \text { (O-rich) }\end{array}$ & \\
\hline
\end{tabular}


The results of defect formation energies are tabulated in Table 2 to compare with other experimental and theoretical values. The result in $O$-rich condition is consistent with the experimental results of formation energy for the Ti interstitial, oxygen vacancy, and Ti vacancy in rutile $\mathrm{TiO}_{2}$.

\section{Defect energy levels}

Since we have adjusted the band gap of $\mathrm{TiO}_{2}$ and the formation energy is in close agreement with the experimental results, the defect induced energy states provide us with a reliable result from the DOS calculation. The partial DOS calculation of the interstitial $\mathrm{Ti}\left(T i_{i}^{i g}\right)$ in the supercell structure $\left(\mathrm{Ti}_{17} \mathrm{O}_{32}\right)$ [Fig. 4] shows the Ti $3 d$-like defect states formed at $1.23 \mathrm{eV}$ below the edge of the conduction band, $E_{c}$. This value is inserted in the energy level diagram of Fig. 1(b) to compare with the experimental results of Ghosh et al. An extra peak $\left(O_{d}\right)$ at $2.14 \mathrm{eV}$ below $E_{c}$ arises due to the octahedral distortion around the interstitial $\mathrm{Ti}$ atom.

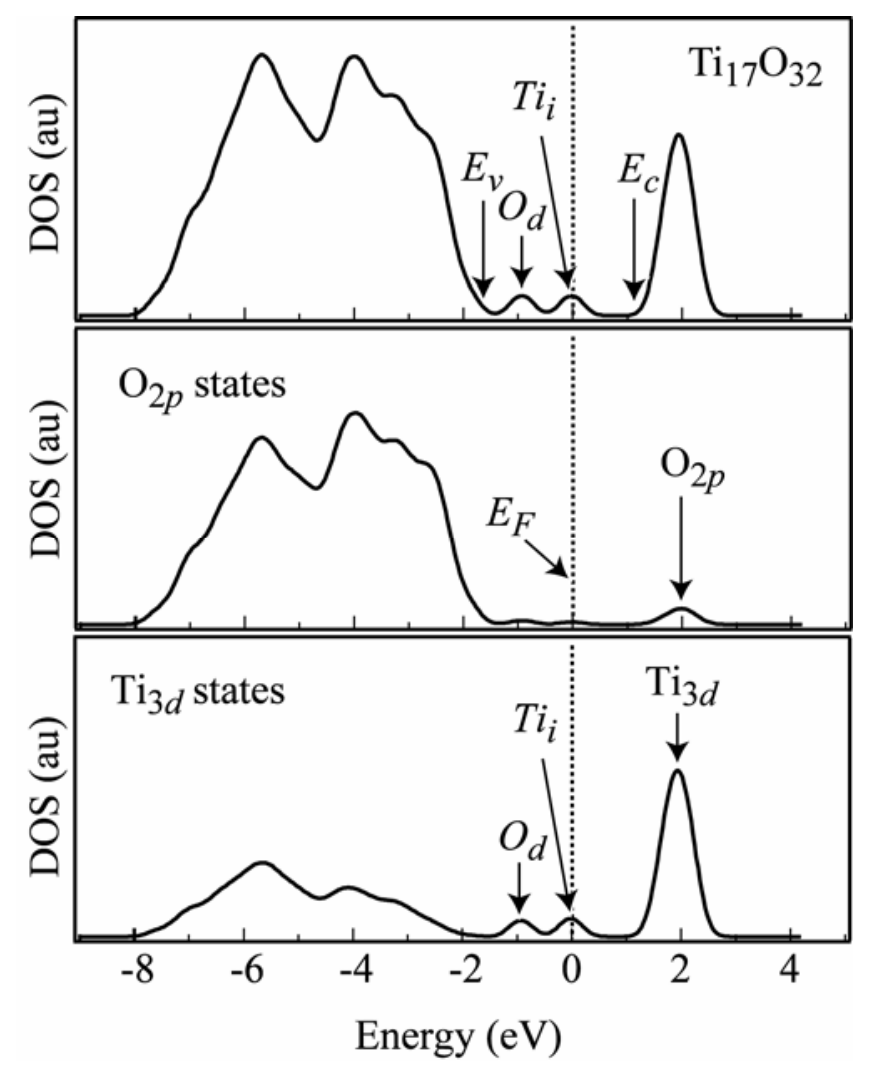

Fig. 4: Interstitial titanium induced density of states (DOS) in bulk rutile $\mathrm{TiO}_{2}$. Zero energy indicates the Fermi level.

The partial DOS calculation of the doubly ionized oxygen vacancy ( $\left.V_{o}^{\mathrm{g}}\right)$ in the supercell structure $\left(\mathrm{Ti}_{16} \mathrm{O}_{31}\right)$ [Fig. 5] shows the Ti 3d-like defect states formed at $1.17 \mathrm{eV}$ below the edge of the conduction band, $E_{c}$ and the Fermi level is pinned by the defect states. The value of this energy level is inserted in Fig. 1(a) to compare with the experimental results of Cronemeyer. It is worth mentioning here that the underestimated band gap was adjusted to match and the calculated formation energy is in fair agreement with the experimental results. Hence, the oxygen vacancy induced defect state at $1.17 \mathrm{eV}$ below $E_{c}$ is reliably a correct measure. 


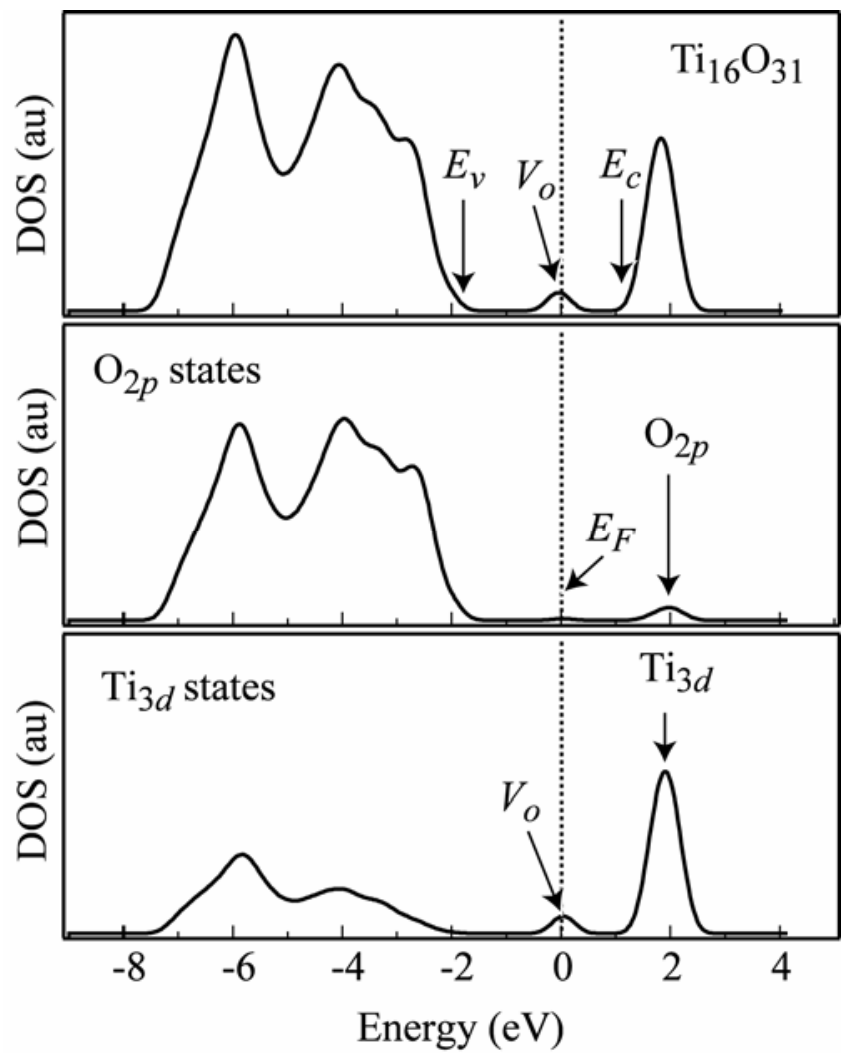

Fig. 5: Oxygen vacancy induced density of states (DOS) in bulk rutile $\mathrm{TiO}_{2}$. Zero energy indicates the Fermi level.

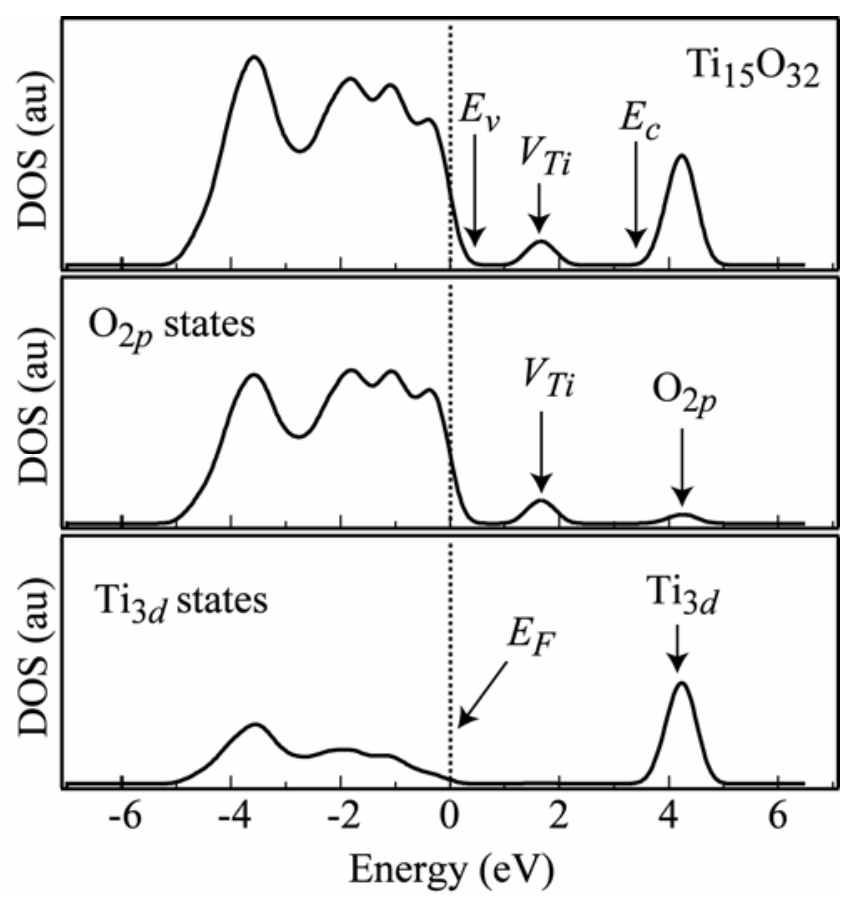

Fig. 6: Titanium vacancy induced density of states (DOS) in bulk rutile $\mathrm{TiO}_{2}$. Zero energy indicates the Fermi level. 
The partial DOS calculation of the Ti vacancy in the supercell structure $\left(\mathrm{Ti}_{15} \mathrm{O}_{32}\right)$ [Fig. 6] shows the Ti vacancy induced $\mathrm{O} 2 p$-like defect states formed at $1.15 \mathrm{eV}$ above the edge of valence band, $E_{v}$. Hence, the Ti vacancy captures electrons from its surrounding $\mathrm{O}$ atoms and makes acceptor-like defect states in the lower half of the band gap. We illustrate this result in order to compare with $V_{o}^{\text {g }}$ and $T i_{i}^{\text {gg }}$ induced energy levels in Fig. 1(b). Unfortunately, no such values are available in the literature for comparison. Since we have achieved a reliable value of the formation energy of a $\mathrm{Ti}$ atom in this system, our calculated $\mathrm{Ti}$ vacancy induced defect energy level is probably acceptable.

In summary, our calculated results for the doubly ionized oxygen vacancy, Ti interstitial, and Ti vacancy are tabulated in Table 1 to compare with other theoretical values. It is clear from the comparison that this theoretical result best reproduces the experimental observations illustrated in Fig. 1 than the other theoretical results summarized in Table 1.

\section{Discussion}

There are many difficulties in detecting the exact position of defect energy levels in a real system using the experimental results on account of the complications for determining the defect structure. There have been debates on the subject of the dominance of Ti interstitials or oxygen vacancies in moderate to highly reduced $\mathrm{TiO}_{2}$. Even a desirable perfect crystal of rutile $\mathrm{TiO}_{2}$ shows a slightly reduced nature even under well-controlled preparation conditions. Hence, the determination of defect types and the energetic position of defect induced energy level are complicated both from experimental and theoretical viewpoints. In this paper we have tried to throw some light on this topic.

The present calculations indicate that the oxygen vacancy and titanium interstitial are donor-like defects, because the induced energy levels form in the upper part of the band gap and the titanium vacancy is an acceptor-like defect due to the formation of an induced energy level at the lower part of the band gap. As we observe from Fig. 4 and 5 that the defect induced energy levels are occupied upon pinning the Fermi level, the result indicates that the defect induced carries are localized in such a manner that both $T i_{i}^{\bullet \bullet}$ and $V_{o}^{\bullet \bullet}$ defects form Ti $3 d$-like energy states inside the upper-half of the band gap. On the other hand, Fig. 6 shows that the Ti vacancy forces the Fermi level towards the valence band, capturing electrons from its surrounding $\mathrm{O}$ atoms to make acceptor-like defect states. It is also confirmed that the scissors operator has no shifting effects on $V_{T i}$ induced states i.e. it has no interactions with other $\mathrm{Ti}$ atoms in the structure.

We have identified the critical problems associated with detecting the absolute position of defect energy levels for different types of point defects. Those problems are two-fold: first, the defect formation energy, which is related to the chemical potential of individual species in the compound, and second, the limitations of the DFT (LDA-GGA) approximation on the band gap energy. Although it is difficult to calculate the absolute value of chemical potential of $\mathrm{Ti}$ and $\mathrm{O}$ in the Ti-O system, our limiting values of the chemical potentials in Ti-rich and O-rich conditions give reasonable estimates of the formation energies. Comparison with the experimental results in Table 2 confirm the suitability of $\mathrm{O}$-rich condition in the $\mathrm{TiO}_{2}$ system. Since we have no experimental results available for the formation energy of a Ti vacancy, the reliability of this estimate is not confirmed at this stage.

In this calculation, we have considered only the charge neutral structure by changing oxidation states (charge states) adjacent to the neutral point defects and formulated the equations for the 
formation energies devoid of chemical potentials of electrons. As a consequence, we were unable to study a charge biased defect structure of singly ionized oxygen vacancy $\left(V_{o}^{\bullet}\right)$.

\section{Conclusion}

The focus of this paper has been on the effect of oxygen nonstoichiometry and the related defect structure on the band gap width and the local energy levels of the lattice point defects. The formation energies and the position of energy levels for bulk point defects (Ti interstitials, oxygen vacancies, and Ti vacancies) inside the bulk band gap of rutile $\mathrm{TiO}_{2}$ have been calculated. Apart from those of the Ti vacancies, our calculated results on the formation energy are in fair agreement with the experimental results and the defect energy levels consistently support the experimental observations. The calculated results indicate that the exact position of defect energy levels depends on the estimated band gap and also the charge state of the point defects of $\mathrm{TiO}_{2}$.

These theoretical results provide fundamental data for further calculations on how the isolated energy bands form and/or how the band gap can be reduced via the interaction of intrinsic defect complexes. Since we have considered only the charge neutral structure in this work, future work may also be concerned with charged point defects.

\section{Acknowledgements}

This work has been supported by the Australian Research Council under the Discovery Project Grants Scheme. One author (LS) gratefully acknowledges the support of the Australian Institute of Nuclear Science \& Engineering.

\section{References}

[1] A. Fujishima and K. Honda, Nature (London), 37 (1972) p.238.

[2] A. L. Linsebigler, G. Q. Lu, J. T. Yates, Chem. Rev., 95 (1995) p.735.

[3] T. Bak, J. Nowotny, M. Rekas and C. C. Sorrell, Int. J. Hydrogen Energy 27 (2002) p.991.

[4] S. U. M. Khan, M. Al-Shahry, and W. B. Ingler Jr., Science, 297 (2002) p.2243.

[5] D. C. Cronemeyer, Phys. Rev.,113 (1959) p.1222.

[6] A. K. Ghosh, F. G. Wakim, and R. R. Addiss, Jr., Phys. Rev., 184 (1969) p.979.

[7] S. Munnix and M. Schmeits, Solid State Commun., 50 (1984) p.1087.

[8] M. Ramamorthy, R. D. King-Smith, and D. Vanderbilt, Phys. Rev. B, 49 (1994) p.7709.

[9] N. Yu and J. W. Halley, Phys. Rev. Lett., 51 (1995) p.4768.

[10] J. P. Perdew, K. Burke, and M. Ernzerhof, Phys. Rev. Lett., 77 (1996) p.3865.

[11] D. Vanderbilt, Phys. Rev. B, 41 (1990) p.7892.

[12] M. C. Payne, M. P. Teter, D. C. Allan, T. A. Arias, and J. D. Joannopoulos, Rev. Mod. Phys., 64 (1992) p.1045.

[13] L. J. Clarke, I. Stich, and M. C. Payne, Comput. Phys. Commun., 72 (1992) p.14.

[14] K. S. Forland, Tionde Nordiske Kemistmotet, Stockholm (1959).

[15] K. S. Forland, Acta Chem. Scand., 18 (1964) p.1267.

[16] P. Kofstad, J. Phys. Chem. Solids, 23 (1962) p.1579.

[17] J-F Marucco, J. Gautron, and P. Lemasson, J. Phys. Chem. Solids, 42 (1981) p.363.

[18] J. R. Akse and H. B. Whitehurst, J. Phys. Chem. Solids, 39 (1978) p.457.

[19] H. Sawatari, E. Iguchi, and R. J. D. Tilley, J. Phys. Chem. Solids, 43 (1982) p.1147.

[20] R. N. Blumenthal, J. Coburn, J. Baukus, and W. M. Hirthe, J. Phys. Chem. Solids, 27 (1966) p.643. 
Defects and Diffusion Ceramics Abstracts

doi:10.4028/3-908451-28-0

The Effect of Defect Disorder on the Electronic Structure of Rutile TiO<sub>2$\mathbf{x}</$ sub $>$

doi:10.4028/3-908451-28-0.1 